\title{
Avoiding the Thorns of the Gifted Red Rose: Case Report of Late Diagnosis of Polycythemia Rubra Vera in a Sibling Bone Marrow Transplantation Donor for a Patient with Chronic Myeloid Leukemia
}

\author{
Irina Amitai ${ }^{a}$ b Jeffrey H. Lipton ${ }^{c}$ \\ a Division of Hematology, Odette Cancer Centre, Sunnybrook Health Sciences Centre, Toronto, ON, Canada; \\ ${ }^{b}$ Sackler Faculty of Medicine, Tel Aviv University, Tel Aviv, Israel; ' Allogeneic Blood and Marrow Transplant Service, \\ Princess Margaret Cancer Centre, Toronto, ON, Canada
}

Dear Editor,

A 25-year-old man presented in 1995 with Philadelphia chromosome-positive chronic myeloid leukemia (CML). He underwent allogeneic hematopoietic stem cell transplantation using his fully-matched 28 -year-old brother as a donor, and eventually was cured. He remains with undetectable disease as measured by PCR, sensitive to greater than 5-log.

Twenty-one years later, the donor was diagnosed with JAK2 exon-12-positive polycythemia rubra vera, with normal cytogenetics. Molecular testing was negative for BCR-ABL. A review of his bloodwork and a bone marrow examination done at the time of his stem cell donation showed no morphologic abnormalities. Cytogenetic or molecular testing was not done at that time. His own stem cells, hosted by his sibling, show no sign of a myeloid disorder and do not carry the JAK2 mutation.

Myeloproliferative neoplasms (MPNs) are sporadic in nature, but familial clustering exists. Presumably, a germline predisposition facilitates the gaining of a somatic driver mutation (JAK2, CALR, and MPL) leading to an MPN phenotype [1]. A large population-based study showed that first-degree relatives of MPN patients have a 5- to 7-fold increased risk of any MPN and a borderline 2-fold increased risk of CML compared with controls [2]. An Italian study reported a prevalence of $7.6 \%$ in familial cases within a population of patients with MPN [3]. The coexistence of CML and other MPNs in the same family has been further reported, occurring less commonly than $2 \mathrm{Ph}$-negative MPNs [1-3]. The coexistence of JAK2, CALR, and MPL somatic mutations in 3 relatives of the same pedigree has also been reported [3].

Significant evidence for a germline predisposition to MPN came from the discovery of a particular JAK2 haplotype in chromosome 9p (the GGCC or 46/1 haplotype), which seems to preferentially acquire the JAK2-V617F mutation. Either this inherited haplotype is genetically unstable and acquires the mutation faster ("hypermutability hypothesis"), or the JAK2-V617F mutation occurs at either $46 / 1$ or the wild haplotype at an equal rate, but there is a selective advantage to the $\mathrm{V} 617 \mathrm{~F}$ clone on the inherited 46/1 haplotype ("fertile ground hypothesis") [3, 4].

Another study on a cohort from Iceland identified a germline polymorphism of the
TERT gene [1]. This TERT variant was associated with increased proliferation of myeloid cells and exerted a similar risk of either MPN.

Other genetic factors have been described lately. These include single nucleotide polymorphisms within JAK2 (such as the ones described above) and the erythropoietin receptor [2] and germline variants that may enhance hematopoietic progenitor differentiation and acquisition of driver mutations (ATG2B and GSKIP duplication, RBBP6 mutations, and SH2B3 [LNK] mutations) $[1,3]$.

Transmission of donor-derived clones through transplantation has been reported [5]. In our case, the overt donor clone developed years after the harvesting and only in the donor's bone marrow.

Screening donors with bone marrow aspirates or extensive genome sequencing as part of the pretransplant evaluation is debatable. Allogeneic hematopoietic stem cell transplantation is often the only treatment option. The identification of latent or constitutional genetic abnormalities subjects healthy donors to potential anxiety and its effect on the clinical outcome is unclear.
Irina Amitai

Division of Hematology, Odette Cancer Centre

Sunnybrook Health Sciences Centre, 2075 Bayview Avenue TG 260

Toronto, ON M4N 3M5 (Canada)

E-Mail irinayoav@gmail.com 


\section{Statement of Ethics}

No institutional or national ethics committee approval was needed. Every effort was made to preserve the patient's confidentiality.

\section{References}

1 Rumi E, Cazzola M. Advances in understanding the pathogenesis of familial myeloproliferative neoplasms. Br J Haematol. 2017 Sep; 178(5):689-98.

2 Landgren O, Goldin LR, Kristinsson SY, Helgadottir EA, Samuelsson J, Björkholm M. Increased risks of polycythemia vera, essential thrombocythemia, and myelofibrosis among

\section{Disclosure Statement}

The authors have no financial conflict of interest to declare.

24,577 first-degree relatives of 11,039 patients with myeloproliferative neoplasms in Sweden. Blood. 2008 Sep;112(6):2199-204.

3 Tashi T, Swierczek S, Prchal JT. Familial MPN predisposition. Curr Hematol Malig Rep. 2017 Oct;12(5):442-7.

4 Jones AV, Chase A, Silver RT, Oscier D, Zoi $\mathrm{K}$, Wang $\mathrm{YL}$, et al. JAK2 haplotype is a major

\section{Author Contributions}

Both authors contributed to the writing of this paper.

risk factor for the development of myeloproliferative neoplasms. Nat Genet. 2009 Apr; 41(4):446-9.

5 Aikawa V, Porter D, Luskin MR, Bagg A, Morrissette JJ. Transmission of an expanding donor-derived del(20q) clone through allogeneic hematopoietic stem cell transplantation without the development of a hematologic neoplasm. Cancer Genet. 2015 Dec;208(12):625-9. 